
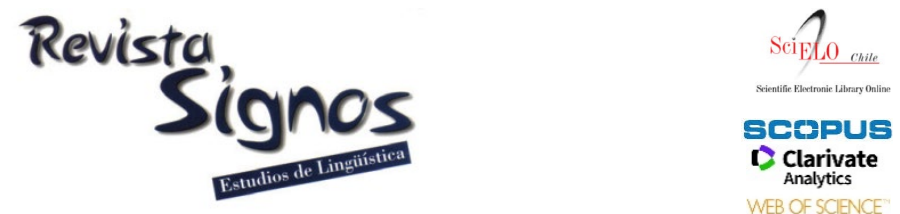

AFB OF SCIFNCE

\title{
Influencia de los estereotipos de género en la comprensión de oraciones. Implicancias para los modelos basados en restricciones
}

\author{
Influence of gender stereotypes in sentence comprehension. \\ Implications for constraint-based models
}

\author{
Nicolás Acuña Luongo \\ UNIVERSIDAD AutóNOMA DE CHILE \\ CHILE \\ acuna.nicolás17@gmail.com
}

\author{
Catherine Elisa Adames Valencia \\ UNIVERSIDAD ANDRÉS BELLO \\ CHILE \\ c.adames.valencia@gmail.com
}

Recibido: 08-IX-2019 / Aceptado: 08-IV-2020
DOI: $10.4067 /$ S0718-09342021000100006

\section{Resumen}

Presentamos una investigación respecto del procesamiento sintáctico-oracional mediante técnicas de medición de movimientos oculares que se enmarca en torno al debate de los modelos basados en restricciones de índole conexionista. El objetivo es determinar si los estereotipos de género, en tanto creencias compartidas, influyen en el procesamiento sintáctico oracional. Participaron 35 estudiantes universitarios quienes leyeron oraciones en las que, en un caso, se cumplían los estereotipos de género y, en otro, se violaban. Todas las oraciones tenían la estructura: sujeto $(\mathrm{S})+$ verbo $(\mathrm{V})+$ objeto directo $(\mathrm{OD})+$ objeto indirecto $(\mathrm{OI})+$ adjunto ('El padre le compró un vestido a su hija/o para su cumpleaños'). El OD corresponde a un sintagma nominal que, en conjunto con $\mathrm{S}$, sugieren un estereotipo de género. En OI, el destinatario variaba de género gramatical en cada caso, de este modo, cumpliendo o violando el estereotipo de género. Se encontraron diferencias significativas en la duración total de fijaciones de cada tipo de oración. Además, hubo diferencias significativas en las regresiones desde OI hacia OD. Esto sugiere que se produce una interrupción del procesamiento incremental cuando los lectores leen el OI que viola el estereotipo provocando una relectura de OD con el objetivo de reconfirmar la interpretación inicial. Los resultados apoyan los modelos basados en restricciones más allá de una perspectiva lexicalista, puesto que el conocimiento general de mundo sí influye en la construcción de la interpretación sintáctica.

Palabras Clave: Procesamiento oracional, estereotipos de género, movimientos oculares, modelos de procesamiento sintáctico. 


\begin{abstract}
We present an investigation on syntactic parsing by means of eye tracking techniques, which is framed around the debate of the restriction-based models of connectionist nature. The objective is to determine if gender stereotypes, as shared beliefs, influence syntactic parsing. The participants were 35 university students, who read sentences in which, in one condition, gender stereotypes were fulfilled and in the other condition, they were violated. All the sentences had the structure: subject $(\mathrm{S})+\operatorname{verb}(\mathrm{V})+\operatorname{direct}$ object (DO) + indirect object (IO) + adjunct (El padre le compró un vestido a su hija/ o para su cumpleaños). The DO corresponds to a noun phrase that, in conjunction with $\mathrm{S}$, suggest a gender stereotype. In $\mathrm{IO}$, the addressee varied in grammatical gender in each case, thus, fulfilling or violating the gender stereotype. Significant differences were found in the total fixation duration of each type of sentence. In addition, there were significant differences in regressions from IO to DO. This suggests that there is an interruption of incremental processing when readers read the $\mathrm{IO}$ that violates the stereotype causing a rereading of DO in order to reconfirm the initial interpretation. The results support constraint-based models beyond a lexicalist perspective, since general knowledge of the world does influence the construction of syntactic interpretation.
\end{abstract}

Key Words: Sentence comprehension, gender stereotypes, eye movements, syntactic parsing models.

\title{
INTRODUCCIÓN
}

Uno de los aspectos fundamentales en la comprensión del lenguaje corresponde a la construcción de la interpretación sintáctica de la oración que se esté leyendo o escuchando en un momento dado. Este proceso se encuentra en una etapa intermedia entre, por un lado, la decodificación fonológica y léxica y, por otro, la integración semántico discursiva (Pickering \& van Gompel, 2006). El análisis sintáctico (syntactic parsing) es el proceso bajo el cual las personas asignan categorías sintácticas a una palabra o a un grupo de palabras durante el proceso de comprensión (Mitchell, 1994). La descripción de cómo las personas asignan categorías sintácticas a las palabras de una oración supone una serie de retos en términos de cómo este fenómeno se incrusta y relaciona con los demás procesos de comprensión del lenguaje.

Los modelos cognitivos pioneros que explicaron el análisis sintáctico adoptaron una perspectiva modular del procesamiento del lenguaje ${ }^{1}$ (Frazier \& Fodor, 1978; Fodor, 1983). No obstante, una serie de resultados experimentales han cuestionado la perspectiva estrictamente modular respecto del análisis sintáctico. Contrario a lo expuesto por el modularismo, existe importante evidencia de que las operaciones de interpretación sintáctica se ven afectadas por el contexto discursivo (Altmann, Garnham \& Henstra, 1994; Ni, Crain \& Shankweiler, 1996) y aspectos de la información léxico-semántica (Pickering \& Traxler, 1998; Clifton, Traxler, Williams, Mohammed, Morris \& Rayner, 2003). Esta evidencia ha motivado a Traxler, Hoversten y Trevor (2018) a sostener que el análisis sintáctico no constituye una etapa intermedia, desde una perspectiva serial, entre procesos léxicos y discursivos, sino que 
correspondería a un sistema de interfase que media entre la información léxica, oracional y la semántica discursiva. Esta mediación sería una consecuencia de las interacciones entre dichos sistemas interconectados.

Estos resultados experimentales motivaron a los científicos a diseñar modelos alternativos frecuentemente denominados modelos interactivos o modelos basados en restricciones (debido a su influencia conexionista). Los modelos basados en restricciones (Trueswell, Tanenhaus \& Garnsey, 1994; Pickering \& Traxler, 1998; Clifton et al., 2003; Traxler et al., 2018) sostienen que toda la información disponible y el conocimiento previo es utilizado para ponderar las potenciales interpretaciones a lo largo del procesamiento. Además, son paralelos en la medida en que los procesos semánticos y pragmáticos ocurren de forma simultánea al análisis sintáctico influenciándose mutuamente. Adicionalmente, en los modelos basados en restricciones pueden coexistir múltiples interpretaciones en línea que ocurren de forma paralela y probabilística. De este modo, en un momento dado, los lectores construyen múltiples interpretaciones relevantes de un imput dado, y estas interpretaciones son evaluadas probabilísticamente a partir del peso de sus conexiones en una red de tipo conexionista. Esto contrasta con los modelos modulares cuya naturaleza es serial (no interactiva). La interpretación final dependerá del conjunto de restricciones que provea la información semántica, pragmática y sintáctica. Finalmente, algunos modelos basados en restricciones (Elman, 1990) incluyen la anticipación o la expectativa de la estructura y del contenido durante el procesamiento de una oración.

En sus inicios los modelos basados en restricciones sostuvieron que cualquier tipo de información contextual que fuera relevante para la interpretación de una oración es computada en etapas tempranas durante el proceso (Tyler \& Marslen-Wilson, 1977). Sin embargo, esta idea es vaga desde un punto de vista teórico. Tanenhaus y Trueswell (1995) señalaron que los modelos basados en restricciones estaban subespecificados en distintos aspectos. Como respuesta a esta crítica, McRae y Matsuki (2013) sostienen que un objetivo de los modelos basados en restricciones es especificar qué tipo de restricciones se utilizan en contextos particulares para la interpretación de oraciones y cuál es el peso de la influencia de estas restricciones específicas. De hecho, con el advenimiento de la perspectiva modular ${ }^{2}$, las investigaciones más recientes sobre la interpretación sintáctica se han centrado en la relación que existe entre el analizador sintáctico y los sistemas cognitivos relacionados (procesamiento léxico y discursivo). Lo que se busca es precisar hasta qué punto y de qué manera el análisis sintáctico se ve influenciado por las demás etapas del procesamiento lingüístico y por el conocimiento de mundo. Diversos modelos interactivos (MacDonald, Pearlmutter \& Seidenberg, 1994; Trueswell et al., 1994) son lexicalistas en el sentido en que señalan que son las propiedades del léxico las que determinan la construcción la interpretación sintáctica. Sin embargo, existen posturas que señalan que aspectos como la prosodia (Speer \& 
Blodgett, 2006), el contorno visual de una situación comunicativa (Tanenhaus, SpiveyKnowlton, Eberhard \& Sedivy, 1995) o el conocimiento general de mundo (Pickering \& Traxler, 1998) pueden determinar también a la construcción del análisis sintáctico. Nuestra investigación pretende contribuir a desentrañar este problema.

El objetivo de esta investigación es determinar si el sistema de creencias en torno a los estereotipos de género puede o no influir en la construcción de la interpretación sintáctica. Es decir, intentamos responder la siguiente pregunta ¿Pueden las creencias de cada individuo en torno al rol del hombre y la mujer en nuestra sociedad ejercer algún tipo de influencia en el análisis sintáctico? La selección de los estereotipos de género se justifica en la medida en que estos estereotipos se inscriben en el conjunto de creencias del mundo de forma general y exceden las propiedades léxicas que tradicionalmente se han esbozado como fuente de influencia e interacción con el análisis sintáctico. En el caso de que la respuesta sea afirmativa, esto motivaría a pensar que las propuestas lexicalistas son insuficientes en la medida en que no incluyen aspectos que están fuera de las propiedades léxicas de las palabras, pero que sí ejercen influencia en la interpretación sintáctica.

Para llevar a cabo nuestro objetivo, primero presentaremos los principales modelos teóricos basados en restricciones y luego las investigaciones psicolingüísticas vinculadas a los estereotipos de género. Luego, presentaremos nuestra investigación empírica basada en la lectura comprensiva de una serie de textos que comprometen los estereotipos de género. Junto a la lectura, se medirá la conducta ocular de cada uno de los participantes del experimento con el fin de analizar las diferencias entre los patrones oculares de lectura en los distintos textos que presentan situaciones de cumplimiento y violación de los estereotipos de género. Finalmente, se presentarán los resultados de la investigación y su implicancia en el debate de los modelos basados en restricciones.

\section{Modelo basado en la frecuencia de estructuras sintácticas, modelo de flujo dual y modelos de intepretación mínima suficiente}

Dentro del abanico de propuestas interactivas relacionadas con el analizador sintáctico, existen una serie de modelos que pretenden describir de qué manera éste se construye. Una de las propuestas más desarrolladas corresponde a los modelos basados en la frecuencia de estructuras (Levy, 2008; Hale, 2011; Jaeger \& Snider, 2013). Las investigaciones en esta perspectiva se han centrado en el grado en el cual los procesos de análisis descansan en conceptos vinculados con información almacenada respecto de la frecuencia relativa de las distintas estructuras sintácticas. La noción fundamental que subyace a esta perspectiva es que las estructuras sintácticas están insertas en una dimensión continua que va desde las estructuras más frecuentes a las menos frecuentes. El análisis sintáctico se construye de acuerdo con la activación 
de un análisis estructural que es más frecuente en un sentido probabilístico. Sin embargo, esta perspectiva requiere de un desarrollo más preciso y de una especificación en torno a cómo el analizador computa la frecuencia en virtud de las posibles restricciones de distinto dominio (Mitchell, 1987). Esta dificultad ha sido bautizada como el problema del calibre del grano (grain size problem). Siguiendo a Traxler et al. (2018), el problema consiste en que los diferentes niveles de análisis pueden producir distintos patrones de preferencia. Si adoptamos un análisis lingüístico amplio de las estructuras sintácticas podemos asumir que, a pesar de las palabras específicas de una oración, los verbos frecuentemente aparecen con un objeto directo. En un sentido más fino y restringido, un verbo particular es probable que frecuentemente aparezca sin un objeto directo. En un nivel incluso más fino, algunos verbos que frecuentemente no tienen objeto directo podrían tenerlo en virtud de ítems léxicos específicos. Traxler et al. (2018) sostienen que este problema aún no ha sido resuelto de forma satisfactoria en el sentido en que todavía no se ha establecido qué información estadística exacta está representada en la memoria ni en qué nivel de análisis (más amplio, más estrecho). Tampoco hay información certera respecto de cómo el sistema de procesamiento lingüístico pondera la información de los distintos niveles en una situación determinada.

Otro modelo basado en restricciones corresponde al modelo de flujo dual (MacDonald et al., 1994; Trueswell, 1996; Trueswell, Tanenhaus \& Kello, 1993). Según esta perspectiva, las palabras aisladas y las estructuras sintácticas guían el procesamiento interpretativo y ambas son fuentes de información para la construcción de significado. En estos modelos la asignación del parsing sintáctico y/o la resolución de ambigüedad sintáctica es similar a, y depende de la resolución de ambigüedad léxica. Además, esta perspectiva señala que los significados que son derivados exclusivamente desde el nivel léxico pueden entrar en conflicto con las interpretaciones sintácticamente inducidas. Esta idea fue apoyada por resultados experimentales respecto de la comprensión de oraciones de vía muerta. Trueswell y colaboradores (1994) examinaron las fijaciones oculares en oraciones como las siguientes:

(1) The defendant examined by the lanyer turned out to be unreliable

[El acusado investigado por el abogado resultó no ser confiable]

(2) The evidence examined by the lanyer turned out to be unreliable.

[La evidencia examinada por el abogado resultó no ser confiable]

El efecto de vía muerta se deriva de la ambigüedad del término examined, que en inglés puede funcionar como forma pasado del verbo 'examinar' o como participio 'examinada'. Debido al principio de inmediatez (Just \& Carpenter, 1980), cuando las personas leen la palabra examined en el caso de (1) tienden a interpretarla como verbo principal de la oración y no como participio. Al continuar leyendo la oración se produce el efecto de vía muerta debido a la cláusula de relativo reducida y, de este 
modo, el verbo principal debe ser reasignado a la expresión turned out. A pesar de que las oraciones (1) y (2) son idénticas en aspectos sintácticos y proposicionales, ellas no fueron leídas del mismo modo. El efecto de vía muerta no ocurrió en (2) y la palabra examined fue inmediatamente interpretada como participio. Estas conclusiones se realizaron en virtud del análisis de la duración de fijaciones oculares y regresiones. La diferencia en la duración de fijaciones oculares es interpretada como un indicio en la dificultad de procesamiento cognitivo. En el caso de (1) se encontraron fijaciones más duraderas y en mayor número en la zona de interés examined y, adicionalmente, mayores regresiones que en el caso de (2). Las regresiones em (1) ocurrían desde el área de interés del verbo turned out hacia el participio examined. Como consecuencias, la refijaciones de examined tuvieron mayor duración que en el caso de (2). Trueswell et al. (1994) señalaron que las diferencias se debieron a las propiedades léxicas del sintagma (1) the defendant en oposición con las propiedades léxicas del sintagma de (2) the evidence. En (2) se utiliza un sustantivo inanimado y este tipo de sustantivo no puede funcionar como agente de la acción examinar. Es decir, las propiedades léxicas pueden ejercer influencias en el analizador sintáctico.

La propuesta de Trueswell et al. (1994) se centra en los roles temáticos de los verbos. Los roles temáticos que los verbos asignan a sus argumentos, tales como agente, paciente, instrumento, etc., son considerados por muchas teorías como información almacenada en la estructura argumental de un verbo (Levin \& Rappaport, 1986; Tanenhaus, Carlson \& Trueswell, 1989). Los ejemplos de (1) y (2) se centran en el ajuste temático, es decir, en el ajuste de los sustantivos y los potenciales roles temáticos asignados por los verbos. Diversos estudios han investigado el ajuste temático desde un punto de vista de restricciones de selección binaria, como en el caso de (1) y (2), donde ciertos verbos seleccionan argumentos que requieren que los sustantivos sean animados o inanimados (Caplan, Hildebandt \& Waters, 1994; Clifton et al., 2003). No obstante, otros investigadores han remarcado la idea que los roles temáticos y su ajuste con los sustantivos son dependientes del tipo de evento que exprese (McRae, Ferretti \& Amyote, 1997). Además, esta última perspectiva apunta hacia un continuo de ajuste temático antes que a una naturaleza binaria del tipo sustantivo animado/inanimado para ciertos tipos de estructura argumental. Esto significa que la asignación de roles temáticos no solo se basaría en propiedades léxicosintácticas sino también en propiedades que provienen del conocimiento de mundo que las personas tienen respecto de los roles que entidades específicas u objetos pueden ejercer en distintos tipos de eventos denotados por los verbos.

Ferreira y Patson (2007) plantean una posición más radical denominada modelo de interpretación mínima suficiente (good enough processing). Para estos autores el procesamiento sintáctico no es ejecutado o está subespecificado cuando otros procesos de carácter léxico semántico son suficientes para llegar exitosamente a una interpretación sintáctica. En este sentido, solo se procesan los papeles temáticos que 
son computados en virtud del léxico y la situación expresada. Consideremos las siguientes oraciones:

(3) El arquero de fútbol atrapó la pelota con sus guantes.

(4) El arquero de fútbol atrapó el guante con su pelota.

(5) La pelota atrapó el guante con su arquero de fútbol.

Los resultados de las investigaciones de Ferreira y Patson (2007), sugieren que la lectura de oraciones como (4) o (5) son interpretadas como (3). Es decir, en estos casos ni siquiera el análisis sintáctico se construye en virtud de los inputs de las oraciones (4) y (5). De este modo el procesamiento sintáctico de las unidades de la oración se omite y reemplaza por una interpretación inducida semánticamente. Solo en situaciones determinadas por el contexto particular, las interpretaciones de (4) y (5) se realizan en virtud de los constituyentes y su disposición.

Las investigaciones en el ámbito de la psicolingüística oracional tradicionalmente utilizaron tecnologías que medían los tiempos de reacción a través del método de lectura por ventana y el seguimiento de la trayectoria ocular a través de eye tracking. Sin embargo, desde fines del siglo pasado y crecientemente en esta década se empezaron a realizar estudios electrofisiológicos que miden la actividad cerebral generada en respuesta a ciertos eventos específicos, en términos de los potenciales eléctricos inducidos por dichos eventos (potenciales evocados).

Uno de los potenciales evocados más importantes para el procesamiento lingüístico es el P600, que corresponde a un potencial de acción de polaridad positiva que se observa entre los 600 y 900 ms tras la exposición de un estímulo. Los estudios seminales de Hagoort, Brown y Groothusen (1993) reportaron que el P600 se presenta con una gran amplitud cuando los sujetos leen oraciones con violaciones sintácticas que involucran concordancia.

(6) The spoiled child throw the toys on the floor.

[El niño mimado tiras los juguetes al suelo.]

(7) The woman congratulated himself.

[La mujer se felicitó a sí mismo.]

Los sujetos presentaron una activación del potencial P600 con gran amplitud cuando leían oraciones como (6) y (7). Inicialmente el P600 se interpretó como la contraparte sintáctica del potencial N400, el cual previamente fue descubierto como un potencial sensible a manipulaciones semánticas. El potencial N400 se presenta con mayor amplitud en la medida en que una palabra no se ajusta semánticamente con el texto precedente (Kutas \& Hillyard, 1980). Mientras que el potencial P600 se interpretó como un reflejo de mecanismos de reanálisis estructural o como un mecanismo de reparación de violaciones gramaticales (Kutas, Van Petten \& Kluender, 2006). 
La idea de que el P600 refleja mecanismos exclusiva y específicamente sintácticos ha sido cuestionada por una serie de investigaciones (Kuperberg, Sitnikova, Caplan \& Holcomb, 2003; Kim \& Osterhaut, 2005). En particular, se ha observado un efecto de P600 en respuesta a oraciones sintácticamente gramaticales, pero semánticamente anómalas tales como:

(8) Every morning at breakfast the eggs would eat. (Kuperber et al., 2003)

[Cada mañana al desayuno los huevos comerían.]

(9) The hearty meal was devouring the kids. (Kim \& Osterhaut, 2005)

[La comida abundante estaba devorando a los niños.]

Lo interesante de esta investigación es que se haya encontrado la activación del potencial P600 en oraciones gramaticalmente correctas, pero semánticamente anómalas en términos de roles temáticos. La conclusión a la que llegan Kim y Osterhaut (2005) respecto de la activación del potencial de P600 en oraciones como (8) y (9) es que al menos, bajo ciertas condiciones, la información semántica 'controla' cómo las palabras deben ser combinadas durante el procesamiento de una oración. Dado que la combinatoria corresponde al dominio de la sintaxis, es posible concluir que estos casos revelan la influencia de fenómenos semánticos en la construcción del parsing sintáctico.

En investigaciones recientes se han propuesto una serie de modelos neurocognitivos para explicar estos resultados, incluyendo el modelo de flujo dual (Kuperberg, 2007; Brouwer, Fitz \& Hoeks, 2012). Como se señaló anteriormente este modelo sostiene que la interpretación sintáctica mezcla el análisis de las reglas gramaticales con un analizador semántico el cual combina las palabras con base en sus características semánticas para construir una sólida interpretación inicial de las palabras que se insertan en el input lingüístico a lo largo de la lectura. De acuerdo con el analizador semántico, el contenido de las palabras morning, breakfast, eggs y eat debería ser combinado en un escenario plausible donde los huevos son comidos. En contraste, el análisis sintáctico genera una interpretación que entra en conflicto en la cual los huevos realizan la acción de comer. En diversos modelos, el conflicto que se genera de estos dos flujos es el que produce el P600 (Bornkessel-Schlesewsky \& Schlesewsky, 2008; Van Herten, Chwilla \& Kolk, 2006; van Herten, Kolk \& Chwilla, 2005; Kuperberg, 2007; Kos, Vosse, Van Den Brink \& Hagoort, et al., 2010).

La sección presentada hasta acá tiene como objetivo enmarcar la discusión teórica en torno a la relación entre el grado de autonomía del procesamiento sintáctico y el distinto tipo de información léxica y/o pragmática que puede estar ejerciendo influencia en parsing sintáctico. En la sección de discusión de resultados de la presente investigación estableceremos de qué forma pueden ser interpretados nuestros hallazgos en virtud de los distintos modelos presentados. Es decir, discutiremos qué modelo se ajusta de mejor manera a los resultados reportados. 


\section{Estereotipos de género y procesamiento lingüístico}

Expuesto hasta aquí el debate teórico de la representación sintáctica, a continuación, presentaremos investigaciones que han estudiado la influencia de los estereotipos de género en la comprensión lingüística. Cabe destacar que estos estudios no se han centrado en el debate teórico de la construcción del análisis sintáctico, sino que preferentemente en la comprensión de pronombres y su relación con la construcción del modelo situacional y los estereotipos de género.

Moulton, Robinson y Elias (1978) realizaron un estudio basado en el uso de pronombres masculinos genéricos. En diversas lenguas se utiliza un pronombre típicamente masculino para referir tanto a hombres como mujeres. Moulton et al. (1978) le propusieron a una serie de participantes dos temas (por ejemplo, being a student o being concerned with looks) y junto con estos temas se utilizó el pronombre genérico his para describir a estas personas. Los autores les pidieron a sus participantes que escribieran una historia sobre el personaje ficticio que calzara con estos temas. La mayoría de las veces, los participantes seleccionaron a un personaje de sexo masculino puesto que calzaba tanto con la forma genérica del pronombre como con la forma masculina específica. La utilización de personajes femeninos solo se realizó cada vez que el tema en cuestión involucraba una actividad típicamente asociada a los roles sociales de las mujeres. El problema de este estudio es que no se basa en el procesamiento lingǘstico, sino que solo en la construcción en una historia.

Otros estudios vinculados al procesamiento en línea han utilizado sustantivos de rol (Irmen, 2007). Estos sustantivos son definidos como cualquier nombre que incorpora características utilizadas para describir a una persona o a un grupo de personas u ocupaciones (por ejemplo, dentistas o estudiantes). En muchas lenguas, incluyendo el español estos sustantivos están marcados con el género masculino para su uso estandarizado. De este modo la expresión 'los estudiantes' refiere tanto a hombre como a mujeres. La mayoría de las investigaciones respecto del uso de estos sustantivos han revelado que la representación mental que se construye a partir de estos sustantivos tiene un sesgo hacia los hombres antes que hacia las mujeres. Estos estudios han utilizado paradigmas tales como la lectura por ventana (Irmen \& Roßberg, 2004) y eye tracking (Irmen, 2007).

Una investigación más reciente fue la realizada por Redl, Eerland y Sanders (2018). Los autores estudiaron la influencia de los estereotipos de género en el procesamiento de los pronombres masculinos genéricos y su influencia en el modelo situacional del discurso. Los autores encontraron que no existe un sesgo hacia la representación masculina cuando se utilizan pronombres masculinos genéricos en situaciones en donde los estereotipos de género no se violaban. Consideremos las siguientes oraciones 
(10) Everyone was brushing his teeth. Amber/Robert, too, was getting ready to go to bed.

[Todos estaban lavándose los dientes. Amber/Robert, también, se estaba preparando para ir a la cama]

Redl et al. (2018) no encontraron diferencias en el comportamiento ocular en la variación de los nombres propios Amber/Robert. En este caso no existe, por lo tanto, un sesgo en la representación situacional del pronombre masculino genérico 'bis'. Sin embargo, la situación es distinta en casos como (11) y (12).

(11) Everyone was painting his nails. Amber/Robert was also applying a bright nail polish

[Todos estaban pintándose las uñas. Amber/Robert también estaba aplicando un esmalte de uñas brillante]

(12) Everyone was cleaning his golf clubs. So Amber/Robert was also brushing some dried-up earth from the clubs.

[Todos estaban limpiando sus palos de golf. Así que Amber/Robert también estaba cepillando tierra seca de los clubes.]

En el caso en que el contexto oracional presentaba una situación típica de rol social femenino y luego era acompañada de un nombre propio masculino, Redl et al. (2018) encontraron que la duración de las fijaciones y las regresiones en estos casos era mayor que en (13). Este efecto era menos pronunciado cuando el contexto oracional presentaba una situación típicamente de rol masculino y luego era seguida de un nombre propio femenino. Los resultados de los autores sugieren que la violación del estereotipo masculino tiene mayor costo de procesamiento que la violación del estereotipo femenino. Sin embargo, el estudio de Redl et al. (2018) se centra fundamentalmente en la relación entre estereotipos de género y pronombre masculino neutro en el contexto de la representación situacional de un segmento discursivo. Nuestra propuesta, por el contrario, se centra en la influencia que puedan tener los estereotipos de género en la construcción de la interpretación sintáctica con el objetivo de determinar si los componentes que puedan influir en el analizador pueden exceder los límites de las propiedades léxicas de las palabras en el contexto de los modelos de flujo dual. En el caso de que la respuesta sea positiva, la investigación constituiría evidencia a favor de las propuestas que extienden los factores que influencian el análisis sintáctico más allá de las propiedades léxicas que exigirían algunos verbos en sus argumentos.

\section{Método}

\subsection{Participantes}

Participaron en este estudio 35 estudiantes universitarios (20 mujeres y 15 hombres). El rango etario de la muestra va desde los 19 años hasta los 22. Todos los participantes son estudiantes chilenos cuya lengua materna es el español. 
Adicionalmente, con el objetivo de homogeneizar la muestra, todos los sujetos son estudiantes universitarios de segundo y tercer año de universidad de las carreras de fonoaudiología (28) y de pedagogía en inglés (7). Como criterio de exclusión, se consideró que ningún participante se encontrara en un tratamiento médico que involucrara medicamentos que alteraran el sistema nervioso central ni tampoco que tuvieran problemas de visión que interfirieran con la recopilación de datos y el rendimiento intelectual propio de la tarea realizada en el experimento.

\subsection{Materiales}

Se construyeron 12 pares de oraciones (24 en total), cuya estructura sintáctica fue sujeto $(\mathrm{S})+$ verbo $(\mathrm{V})+$ objeto directo $(\mathrm{OD})+$ objeto indirecto $(\mathrm{OI})+$ circunstancial. El OD corresponde a un sintagma nominal que, en conjunto con $\mathrm{S}$, sugieren un estereotipo de género. En OI, el destinatario variaba de género gramatical en cada caso, de este modo, cumpliendo o violando el estereotipo de género. Adicionalmente a la oración crítica, cada texto tenía una oración adicional de relleno.

Texto condición 1 (cumplimiento de estereotipo)

Juan le compró un vestido a su hija para su cumpleaños. La fiesta sería el próximo viernes.

Texto condición 2 (violación de estereotipo)

Juan le compró un vestido a su hijo para su cumpleaños. La fiesta sería el próximo viernes.

Como se puede observar, la única diferencia entre los dos estímulos es el morfema flexivo de marca de género en el sustantivo núcleo del sintagma nominal cuya función sintáctica es de OI. Esto permite evaluar si los estereotipos de género, en tanto conjunto de creencias respecto de los roles sociales de hombres y mujeres influye en análisis sintáctico. La violación del estereotipo de género en el texto 2 deriva de la relación que existe entre $\mathrm{V}$ - OD - OI, debido a que $\mathrm{V}$ y OD determinan no solo la selección léxica del núcleo de OI (por ejemplo, las propiedades animado/inanimado) sino que, de acuerdo con los estereotipos de género, también determina la selección del género gramatical del núcleo de OI.

Adicionalmente, se construyeron una serie de estímulos distractores, 32 en total, basados en textos de extensión similar a los ítems críticos. Finalmente, cada estímulo fue acompañado de una sencilla pregunta de comprensión cuyo objetivo era determinar si la lectura de los sujetos se llevaba a cabo con atención o no.

\subsection{Diseño y medidas}

El estudio implementó un diseño intrasujetos. Los ítems críticos se presentaban en dos condiciones. La única variación formal en el estímulo correspondió al morfema 
flexivo de marca de género gramatical incorporado al núcleo del sintagma nominal del objeto indirecto. De los 24 ítems críticos, 12 correspondieron a la versión de género masculino y 12 a la versión de género femenino. A su vez, la mitad de los estímulos correspondían a cumplimiento de los estereotipos de género y la mitad a violación del estereotipo de género. Los estímulos se organizaron en dos grupos con el objetivo de su presentación en el experimento. El primer grupo se compuso de 12 oraciones de las cuales 6 correspondieron a la versión con marca de género masculino y 6 con marca de género femenino. El segundo grupo se compuso de 12 oraciones que eran la contrapartida de cada estímulo del grupo uno. Es decir, en el segundo grupo había 6 oraciones con el morfema flexivo masculino y 6 con morfema flexivo femenino. Con el fin de contrabalancear la presentación de los estímulos, se construyeron 3 versiones de presentación de estímulos distintas.

Las medidas de análisis de movimientos oculares que se computaron como medidas dependientes fueron: a) duración de fijaciones (Holmqvist, Nystrom, Andersson, Dewhurst, Jarodzka \& Van de Weijer, 2011), b) número de fijaciones, c) duración de primera fijación, c) duración de regresiones y d) transiciones. La duración de fijaciones corresponde al tiempo total que un sujeto estuvo al interior de un área de interés, incluyendo las relecturas. Esta medida es frecuentemente homologada con el tiempo total de lectura. El número de fijaciones corresponde a la cantidad de fijaciones que un sujeto realizó en un área de interés determinada. La duración de regresiones -en ocasiones denominadas second pass reading- constituye el tiempo de todas las fijaciones de un área de interés que fueron subsecuentes a la primera lectura (Hyönä, Lorch \& Kaakinen, 2002). Las transiciones corresponden a la duración de regresiones desde un área de interés determinada y que provienen de otra área de interés determinada (Hyönä, Lorck \& Rinck, 2003). Esto permite identificar qué partes del texto desencadenan tareas de reprocesamiento en virtud de su complejidad (entre otros motivos). En efecto, las regresiones son consideradas como resultado de problemas cognitivos que ocurren durante la lectura y que deben ser resueltos a través de la relectura de segmentos anteriores. En este sentido el lugar desde donde ocurre la regresión se interpreta como el desencadenante de la dificultad de procesamiento (Parodi, Julio, Nadal \& Burdiles, 2018).

La selección de estas medidas se justifica por tratarse de indicadores apropiados para identificar las dificultades de procesamiento de un texto (Holmqvist et al., 2011; Parodi et al., 2018).

\subsection{Aparato y procedimiento}

Para la grabación de los movimientos oculares se utilizó el equipo Tobii Eye Tracker TX-300. Este equipo es un eye tracker montado junto a un monitor que captura la información ocular a $300 \mathrm{~Hz}$. La precisión del sistema es inferior a 0,5 grados en 
óptimas condiciones. La resolución del monitor para el experimento fue de $1920 \mathrm{x}$ 1080.

Los participantes se sentaron frente al monitor de computador a una distancia de $65 \mathrm{~cm}$ aproximadamente en una habitación aislada ubicada en las dependencias del laboratorio de Lenguaje y Cognición de la Pontificia Universidad Católica de Valparaíso. Inicialmente se realizó un proceso de calibración para sintonizar el eye tracker.

Se les informó a los participantes que leerían una serie de textos en el monitor del computador y que durante este proceso se grabaría su conducta ocular. Las instrucciones planteaban que la lectura debía ser silenciosa y a un ritmo normal. Una vez que leyeran el texto de la pantalla los sujetos debían presionar la tecla espacio para avanzar a la siguiente actividad consistente en una pregunta de comprensión que presentaba 3 alternativas. Los sujetos debían seleccionar la alternativa correcta con el mouse y luego continuar con el siguiente texto. Estas instrucciones fueron desplegadas en el monitor del computador y adicionalmente fueron leídas en voz alta por el experimentador. A continuación, los sujetos realizaron una lectura de prueba con su consecuente pregunta de comprensión. Terminada la prueba el experimentador preguntó si comprendieron las instrucciones. En caso de responder afirmativamente, entonces se comenzaba a realizar el experimento. La sesión total duró alrededor de 30 minutos.

\subsection{Análisis de datos}

Para efectos de diseño del experimento y análisis de datos se utilizó el software Tobii Studio Pro. Adicionalmente, se utilizó un algoritmo programado en JAVA para calcular algunas medidas adicionales que el programa Tobii Studio Pro carecía.

Las áreas de interés que se dibujaron fueron cinco. La primera corresponde al texto íntegro incluyendo la oración crítica y la segunda oración de relleno. La segunda área de interés la comprende toda la oración crítica. La tercera área de interés corresponde a la oración de relleno. El estudio de esta zona de interés se justifica en la medida en que no esperamos ninguna diferencia significativa entre los textos estereotipados y no estereotipados porque en ambos textos la oración de relleno no varía. La cuarta área de interés la denominamos marcador de estereotipo (ME) y la comprende el núcleo del sintagma nominal de OD. La denominación se justifica en la medida en que consideramos que el OD activa el estereotipo de género provocando de esta manera la expectativa del siguiente argumento OI con su correspondiente flexión de género gramatical. La quinta área de interés la denominamos ítem crítico (IT) y la comprende el núcleo del sintagma nominal de OI el cual cumple o viola los estereotipos de género. A continuación, se presenta un esquema con todas las áreas de interés. 


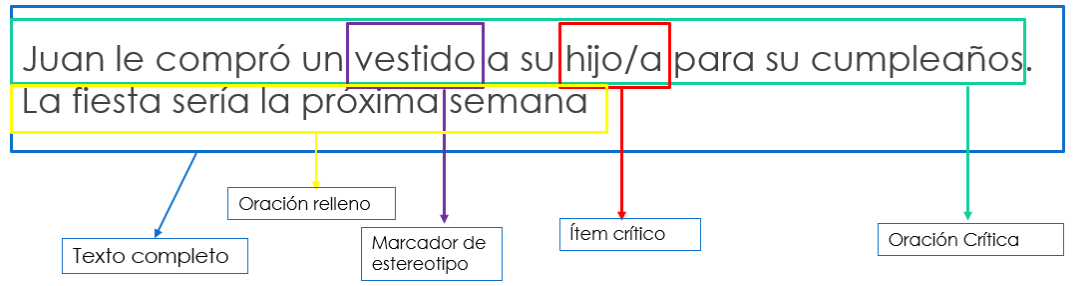

Figura 1. Áreas de interés analizadas.

\section{Resultados}

Los resultados del análisis de datos contemplan la aplicación de la prueba estadística de rangos con signo de Wilcoxon para muestras relacionadas. Esto se debe a que el diseño es de carácter intrasujeto donde la comparación involucra por un lado los datos correspondientes a las lecturas de la condición estereotipada y por otro a la condición no estereotipada. Con respecto al área de interés correspondiente al texto completo (Tabla 1) los datos arrojan que existieron diferencias significativas en el total de fijaciones o tiempo de lectura $(p=.004)$ y en el número total de fijaciones $(p=$ .009). Teniendo en cuenta que la única diferencia entre ambas condiciones corresponde al morfema flexivo de marca de género en el núcleo del sintagma nominal OI, se puede inferir que esta modificación impacta en el procesamiento del texto. Consecuente con el resultado de la medida de tiempo total de lectura, la medida de número total de fijaciones también tuvo una diferencia significativa entre las dos condiciones $(p=.009)$.

Tabla 1. Diferencias entre condición estereotipada y no estereotipada en medidas de tiempo de lectura y número de fijaciones. Área de interés: Texto completo.

\begin{tabular}{|c|l|c|c|c|}
\hline & \multicolumn{4}{|c|}{ Medidas descriptivas y Significancia } \\
\hline \multirow{2}{*}{ Medidas } & \multicolumn{1}{|c|}{ Condición } & $\boldsymbol{M}$ & SD & $\boldsymbol{P}$ \\
\hline \multirow{2}{*}{ Tiempo total de lectura } & Estereotipado & 42840 & 14948 & .004 \\
\cline { 2 - 5 } & No estereotipado & 48130 & 18070 & \\
\hline \multirow{2}{*}{ Número total de fijaciones } & Estereotipado & 220.7 & 64.9 & .009 \\
\cline { 2 - 5 } & No estereotipado & 243.6 & 72.8 & \\
\hline
\end{tabular}

Para determinar que la diferencia entre el tiempo total de lectura se atribuye al cambio en el morfema flexivo de género en el núcleo del sintagma nominal de OI, medimos la conducta ocular en el área de interés de la primera oración, donde se manifiesta la diferencia gramatical entre ambas condiciones. Los resultados (Tabla 2) arrojaron que existe diferencia significativa tanto en la duración total de las fijaciones de la oración crítica $(\phi=.003)$ como en el número total de fijaciones $(\phi<.001)$. Estas diferencias no se encontraron en la oración 2 (Tabla 3), puesto que dicha oración no variaba en ambas condiciones. 
Tabla 2. Diferencias entre condición estereotipada y no estereotipada en medidas de tiempo de lectura y número de fijaciones. Área de interés: Oración crítica.

\begin{tabular}{|c|l|c|c|c|}
\hline & \multicolumn{4}{|l|}{ Medidas descriptivas y Significancia } \\
\hline \multicolumn{1}{|c|}{ Medidas } & \multicolumn{1}{|c|}{ Condición } & $\boldsymbol{M}$ & SD & $\boldsymbol{P}$ \\
\hline \multirow{2}{*}{ Tiempo total de lectura } & Estereotipado & 22258 & 6689 & .003 \\
\cline { 2 - 6 } & No estereotipado & 25070 & 7377 & \\
\hline \multirow{2}{*}{ Número total de fijaciones } & Estereotipado & 114.8 & 44.8 & $<.001$ \\
\cline { 2 - 6 } & No estereotipado & 141.3 & 53.4 & \\
\hline
\end{tabular}

Tabla 3. Diferencias entre condición estereotipada y no estereotipada en medidas de tiempo de lectura y número de fijaciones. Área de interés: Oración 2.

\begin{tabular}{|c|l|c|c|c|}
\hline & \multicolumn{4}{|l|}{ Medidas descriptivas y Significancia } \\
\hline \multicolumn{1}{|c|}{ Medidas } & \multicolumn{1}{|c|}{ Condición } & $\boldsymbol{M}$ & SD & $\boldsymbol{p}$ \\
\hline \multirow{2}{*}{ Tiempo total de lectura } & Estereotipado & 16394 & 4658 & .07 \\
\cline { 2 - 6 } & No estereotipado & 17006 & 4601 & \\
\hline \multirow{2}{*}{ Número total de fijaciones } & Estereotipado & 73.4 & 24 & .15 \\
\cline { 2 - 6 } & No estereotipado & 75.7 & 22 & \\
\hline
\end{tabular}

Para especificar el impacto que tiene la modificación del morfema flexivo entre ambas condiciones, analizamos (Tabla 4) los patrones de lectura en la zona de interés marcador de estereotipo (ME). Debemos remarcar que esta zona de interés no tiene variación entre ambas condiciones. La duración de la primera fijación no tuvo diferencias significativas entre ambas condiciones $(p=.412)$. Sin embargo, se encontró una amplia diferencia significativa en la medida duración total de fijaciones $(p<.001)$ y en el número total de fijaciones $(\not<.001)$ de la misma área de interés. Dado que la primera fijación fue semejante en ambas condiciones, la diferencia en la duración total solo puede atribuirse en virtud de las refijaciones que existieron en dicha zona de interés. Para corroborarlo se analizó la duración total de refijaciones en el área de interés ME (Tabla 4) y se encontraron diferencias significativas entre ambas condiciones $(p=.006)$. Adicionalmente se analizaron las transiciones provenientes desde la zona de interés IC hacia ME. En este caso también se encontraron diferencias significativas entre ambas condiciones $(p=.046)$. Esto sugiere que el comportamiento de lectura de los sujetos se desarrolló normalmente en la condición no estereotipada hasta que leían el ítem crítico. La lectura del ítem crítico desencadenó una serie de regresiones que recayeron sobre $\mathrm{ME}$, en la medida en que la flexión de género de IC violaba el estereotipo de género anticipado por ME. Es decir, al leer el OD de la oración, los sujetos generaron expectativas de lectura que demandaban un determinado morfema flexivo de género para el núcleo de OI. Al no verse cumplidas estas expectativas se generó una dificultad de procesamiento que se manifestó en las regresiones desde IC hacia $\mathrm{ME}$. 
Tabla 4. Diferencias entre condición estereotipada y no estereotipada en medidas de tiempo de lectura y número de fijaciones. Área de interés: Marcador de estereotipo (ME).

\begin{tabular}{|l|l|c|c|c|}
\hline & \multicolumn{4}{|l|}{ Medidas descriptivas y Significancia } \\
\hline \multicolumn{1}{|c|}{ Medidas } & \multicolumn{1}{|c|}{ Condición } & $\boldsymbol{M}$ & SD & $\boldsymbol{P}$ \\
\hline \multirow{2}{*}{ Duración de primera fijación } & Estereotipado & 1605 & 454 & .412 \\
\cline { 2 - 6 } & No estereotipado & 1549 & 316 & \\
\hline \multirow{2}{*}{ Duración total de fijaciones } & Estereotipado & 5722 & 2744 & $<.001$ \\
\cline { 2 - 6 } & No estereotipado & 7890 & 3342 & \\
\hline \multirow{2}{*}{ Número total de fijaciones } & Estereotipado & 25.2 & 7 & $<.001$ \\
\cline { 2 - 6 } & No estereotipado & 35.1 & 10.1 & \\
\hline \multirow{2}{*}{ Duración de refijaciones } & Estereotipado & 1168 & 1121 & .006 \\
\cline { 2 - 6 } & No estereotipado & 1661 & 1370 & \\
\hline \multirow{2}{*}{ Transiciones de IC a ME } & Estereotipado & 153 & 228 & .046 \\
\cline { 2 - 6 } & No estereotipado & 290 & 395 & \\
\hline
\end{tabular}

Por último, se analizaron las medidas de lectura en el área de interés IC. Con respecto a la primera fijación no se encontraron diferencias significativas entre ambas condiciones $(p=.08)$. Este hecho es aparentemente contraintuitivo en la medida en que la lectura de IC en la condición no estereotipada viola las expectativas de lectura. Sin embargo, de acuerdo con los resultados, el costo de procesamiento no recae sobre el elemento detonante demarcado por IC, sino que recae en $\mathrm{ME}$ en el sentido en que la primera fijación de IC demanda inmediatamente una refijación en ME (Tabla 4). Es decir, la variación del morfema flexivo en el núcleo de OI en la condición no estereotipada funciona como desencadenante de reprocesamiento del núcleo de OD, pero sin evidenciar un costo de procesamiento en la propia partícula de OI. Lo anterior se concluye también en virtud de que no existieron diferencias significativas ni en la duración total de fijaciones $(p=.10)$ ni en el número total de fijaciones $(p=.13)$ de IC (Tabla 5). Tampoco se encontraron diferencias significativas en las medidas de regresiones. Ni la duración de refijaciones $(p=.21)$ ni las transiciones de ME a IC ( $p$ $=.37)$ tuvieron diferencias significativas entre ambas condiciones. El hecho de que el área de interés IC no tuviera diferencias significativas, y que, por el contrario, desencadenen fenómenos de reprocesamiento evidenciados a través de regresiones es consistente con otras investigaciones sobre partículas discursivas y conducta ocular (Loureda, Cruz, Rudka, Nadal, Recio \& Zuloaga, 2015; Nadal, Cruz, Recio \& Loureda, 2016; Parodi et al., 2018; Recio, Nadal \& Loureda, 2018). En estas investigaciones los ítems críticos que modifican las condiciones de los estímulos experimentales no involucran un mayor procesamiento en esos ítems, sino que desencadenan regresiones a segmentos que deben ser reprocesados producto de la variación de las partículas discursivas analizadas. 
Tabla 5. Diferencias entre condición estereotipada y no estereotipada en medidas de tiempo de lectura y número de fijaciones. Área de interés: IC.

\begin{tabular}{|l|l|c|c|c|}
\hline & \multicolumn{4}{|l|}{ Medidas descriptivas y Significancia } \\
\hline \multicolumn{1}{|c|}{ Medidas } & \multicolumn{1}{|c|}{ Condición } & $\boldsymbol{M}$ & SD & $\boldsymbol{P}$ \\
\hline \multirow{2}{*}{ Duración de primera fijación } & Estereotipado & 1527 & 420 & .08 \\
\cline { 2 - 6 } & No estereotipado & 1654 & 378 & \\
\hline \multirow{2}{*}{ Duración total de fijaciones } & Estereotipado & 3566 & 2358 & .10 \\
\cline { 2 - 6 } & No estereotipado & 3909 & 2033 & \\
\hline \multirow{2}{*}{ Número total de fijaciones } & Estereotipado & 17 & 8.8 & .13 \\
\cline { 2 - 6 } & No estereotipado & 18 & 7.8 & \\
\hline \multirow{2}{*}{ Duración de refijaciones } & Estereotipado & 1586 & 2.2 & .21 \\
\cline { 2 - 6 } & No estereotipado & 1842 & 1.9 & \\
\hline \multirow{2}{*}{ Transiciones de ME a IC } & Estereotipado & 1308 & 1483 & .37 \\
\cline { 2 - 6 } & No estereotipado & 1423 & 1348 & \\
\hline
\end{tabular}

\section{Discusión}

Los resultados de la presente investigación permiten concluir tentativamente que los estereotipos de género influyen en la construcción de la interpretación sintáctica. No obstante, esta afirmación requiere de justificación. Al respecto, consideramos que la dificultad adicional en la lectura de la condición no estereotípica no constituye un fenómeno exclusivamente semántico, en el sentido en que impacta en la relación que existe entre V-OD-OI. Es decir, los estereotipos de género determinan la selección del morfema flexivo de género gramatical del núcleo del sintagma nominal OI. En este sentido, la relación que se establece entre $\mathrm{V}$ y $\mathrm{OD}$ activa las creencias de nuestros estereotipos de género y esta activación demanda expectativas en torno a qué tipo de morfema flexivo debe presentarse en el núcleo de OI. Para clarificar esta reflexión podemos sostener algunos paralelismos parciales entre los fenómenos de regresiones y transiciones observados en la condición no estereotípica del presente experimento con los reportados por Trueswell (1996) en su estudio de oraciones de vía muerta. Si bien es cierto que en la investigación de Trueswell se trabajó con la ambigüedad estructural de oraciones de vía muerta, el autor encontró que las propiedades léxicas de los sustantivos (animado/inanimado) pueden determinar las interpretaciones sintácticas en oraciones de vía muerta. En los casos de reprocesamiento de la investigación de Trueswell, las regresiones y transiciones ocurrían desde la primera fijación del verbo turned out hacia el verbo examined. Esto ocurría debido a un conflicto con la lectura previa del verbo examined, el cual había sido interpretado inicialmente como verbo principal. De este modo, las transiciones y regresiones sugieren un reprocesamiento en donde la interpretación sintáctica inicial entra en conflicto con el nuevo ítem léxico obligando una reinterpretación del verbo principal. En nuestro estudio no se trabajó con oraciones de vía muerta, pero el efecto es similar en la medida en que el reprocesamiento lo desencadena, en nuestro caso, no un ítem léxico genuino sino un morfema flexivo que entra en conflicto con la hipótesis sintáctica diseñada. Esto provoca una relectura, pero no para construir una nueva representación sintáctica, 
como en el caso de Trueswell (1996), sino que para reconfirmar la hipótesis inicial. Consideramos que el efecto observado no corresponde a un fenómeno semántico en la medida en que la evidencia de la trayectoria ocular exhibe un conflicto entre el núcleo del sintagma nominal de OI y el núcleo del sintagma nominal OD. En este sentido, sugerimos que la activación de los estereotipos de género, activada por la lectura del núcleo de OD, induce una interpretación sintáctica que genera la expectativa de un núcleo de sintagma nominal de OI con un morfema flexivo que sea compatible con la expectativa generada por el estereotipo de género. Cuando esta expectativa es violada, entonces entra en conflicto la relación entre OD y OI. Por ello es que el comportamiento ocular exhibe una mayor duración de fijaciones en el núcleo de OD y no de OI. A su vez las transiciones ocurren desde el núcleo de OI hacia el núcleo de OD. Si el fenómeno fuera exclusivamente semántico, se esperaría que la carga de procesamiento extra estuviera en el ítem léxico que genera el conflicto y no en su relación con las demás estructuras sintácticas. Es último fenómeno el que nos induce a pensar que existe un impacto de los estereotipos de género en el parsing sintáctico. Este impacto no redunda en una interpretación sintáctica alternativa sino en un reprocesamiento para reconfirmar la hipótesis construida inicialmente.

Con respecto a la discusión de los modelos de procesamiento sintáctico, los hallazgos encontrados suponen un problema para el modelo de flujo dual en la medida en que la descripción de los factores que restringen el análisis sintáctico es insuficiente. No solo las propiedades léxicas pueden determinar las interpretaciones sintácticas que se construyen, sino también el conjunto de creencias generales, en nuestro caso los estereotipos de género. La asignación específica de los roles temáticos no solo se basa en propiedades léxico-sintácticas, sino que también en propiedades que provienen del conocimiento de mundo que las personas tienen respecto de los roles que entidades específicas u objetos pueden ejercer en los distintos tipos de eventos denotados por el verbo. Además, la naturaleza de los estímulos utilizados en esta investigación difiere de los tradicionalmente utilizados en este tipo de investigaciones. Frecuentemente se utilizan ítems críticos que varían en su naturaleza léxica generando así diversas consecuencias en el procesamiento (Clifton et al., 2003; Pickering \& Traxler, 1998). En nuestro estudio la diferencia es mucho más sutil, puesto que no se da estrictamente en términos léxicos. Por el contrario, estas diferencias se articulan en torno a la propiedad de género gramatical de los argumentos del verbo. Es decir, no solo podemos sostener que determinados verbos exigen argumentos con determinadas propiedades léxicas, sino que también determinados verbos en conjunto con alguno de sus argumentos pueden determinar las propiedades de género gramatical de núcleo de algún otro argumento, pero no desde una perspectiva de concordancia gramatical, sino que desde una perspectiva basada en el conocimiento general de mundo. De este modo el verbo 'comprar' unido a un objeto directo como 'vestido' puede requerir un argumento de destinatario cuyo género gramatical sea femenino en virtud del 
conocimiento de mundo sobre los estereotipos de género. Sin embargo, debe precisarse que este requerimiento es en términos psicológicos y no en términos estructurales de la lengua. En efecto, nadie juzgaría que una oración que no cumpla con el requisito del morfema flexivo mencionado sea agramatical, sin embargo, mentalmente sí genera procesos semejantes a los que se manifiestan cuando una interpretación sintáctica debe reconstruirse porque resulta ser agramatical, como en el caso de las oraciones de vía muerta.

Nuestros resultados son por una parte concordantes con la propuesta de McRae et al. (1997), quienes sostienen que los roles temáticos y su ajuste con los sustantivos dependen del tipo de evento que se exprese en el verbo y no solamente de las propiedades léxicas-sintácticas que pueda exigir ese verbo en sus argumentos. De este modo, la propuesta del modelo de flujo dual de índole lexicalista es insuficiente para describir el conjunto de factores que determinan el análisis sintáctico. Adicionalmente, nuestros resultados son más concordantes con las propuestas de los modelos basados en frecuencias (Levy, 2008; Hale, 2011; Jaeger \& Snider, 2013). En este sentido, los usuarios del lenguaje generan expectativas respecto de las estructuras sintácticas subsecuentes en virtud de diversos criterios incluidos la frecuencia de las estructuras sintácticas. El efecto de reprocesamiento se deriva del hecho de que la estructura utilizada en el estímulo de condición no estereotipada es poco frecuente. Sin embargo, su baja frecuencia se deriva de aspectos que provienen del conocimiento general de mundo y no de aspectos formales o lexicales.

Con respecto al problema del 'calibre de grano' (grain size problema) consideramos que la probabilidad de aparición de una estructura está condicionada en un continuo que va desde los aspectos puramente gramaticales, pasando por propiedades léxicas y finalizando con propiedades provenientes del conocimiento general de mundo. De este modo, las restricciones más importantes y generales siempre serán aquellas que dominan las reglas estructurales del lenguaje. Un paso más abajo se encuentran las propiedades léxicas que pueden determinar la selección de determinados argumentos para un verbo. En un sentido más fino, el conocimiento de mundo puede influir en la interpretación sintáctica en virtud del tipo de evento denotado por el verbo y/o alguno de sus argumentos. De este modo, el verbo 'comprar' exige un argumento de objeto -nivel estructural-. Además, este verbo demanda en virtud de su frecuencia que el argumento de objeto sea de carácter inanimado -nivel lexical-. Sin embargo, en este nivel la demanda de que aparezca un argumento que seleccione un sustantivo inanimado es menos restrictiva que la de nivel estructural. Es decir, la restricción que impone el nivel estructural en el caso del verbo 'comprar' para la aparición del argumento de objeto es más fuerte que la restricción que demanda que ese objeto sea un sustantivo de carácter inanimado (por ejemplo, contrástese 'comprar un libro' con 'comprar un perro'). En este caso, la exigencia no es obligatoria como en el caso de nivel estructural. Finalmente, en un sentido más fino, el tipo de objeto que sea 
seleccionado como argumento del verbo puede configurar un evento que active conocimiento general de mundo. En este caso, si el argumento objeto del verbo 'comprar' es 'un vestido' el conocimiento general de mundo sobre los roles de hombres y mujeres se activa para restringir el tipo de destinatario que requiere comúnmente ese tipo de evento. Este nivel se proyecta en las propiedades flexivas de género gramatical del núcleo del OI. A pesar de la diferencia en el grado de fuerza que tiene cada nivel que restringe la interpretación sintáctica, la violación de estas restricciones, en cualquier nivel que ocurra (estructural, lexical o de conocimiento general de mundo), genera mecanismos cognitivos semejantes como, por ejemplo, el reprocesamiento evidenciado a partir de las regresiones y transiciones oculares reportadas en los resultados de la presente investigación.

Como futura proyección, consideramos que es necesario realizar un estudio sobre la relación entre potenciales evocados y estereotipos de género. Como ya mencionamos en el marco teórico, la investigación de potenciales evocados puede brindar conclusiones sobre los mecanismos de construcción de interpretación sintácticas. Es pertinente determinar si el reprocesamiento desencadenado por la violación de los estereotipos de género determina una activación de potencial N400 o P600. Esto, porque ya existe evidencia de que en ocasiones la distinción entre un potencial asociado al componente sintáctico como el P600 sea exclusivamente vinculado a este componente. En efecto, el hecho de que componentes semánticos anómalos o poco frecuentes activen en ocasiones el P600 es evidencia de una interacción en línea entre fenómenos sintácticos y semánticos. De acuerdo con los resultados obtenidos en nuestra investigación, sería esperable encontrar una activación en el potencial P600 en la lectura de los estímulos utilizados en este estudio. Sin embargo, esto constituye una hipótesis de investigación que está sujeta a comprobación en investigaciones futuras. Esto aportaría evidencia más concluyente y directa a la hipótesis defendida en este trabajo respecto de la influencia de los estereotipos de género en el parsing sintáctico, específicamente en torno a la selección de las propiedades flexivas de alguno de los componentes de los sintagmas nominales que jueguen determinados roles temáticos que puedan entrar en conflicto con las creencias sobre los roles sociales de los hombres y mujeres.

\section{REFERENCIAS BIBLIOGRÁFICAS}

Altmann, G., Garnham, A. \& Henstra, J. (1994). Effects of syntax in human sentence parsing: Evidence against a structure-based proposal mechanism. Journal of Experimental Psychology: Learning, Memory and Cognition, 20(1), 209-216.

Bornkessel-Schlesewsky, I. \& Schlesewsky, M. (2008). An alternative perspective on "semantic P600" effects in language comprehension. Brain Research Reviews, 59, 55-73. 
Brouwer, H., Fitz, H. \& Hoeks, J. (2012). Getting real about semantic illusions: Rethinking the functional role of the P600 in language comprehension. Brain Research, 1446, 127-143.

Caplan, D., Hildebrandt, N. \& Waters, G. S. (1994). Interaction of verb selectional restrictions, noun animacy and syntactic form in sentence processing. Language and Cognitive Processes, 9(4), 549-585.

Clifton, C. Jr., Traxler, M. J., Williams, R., Mohammed, M., Morris, R. K. \& Rayner, K. (2003). The use of thematic role information in parsing: Syntactic processing autonomy revisited. Journal of Memory and Language, 49, 317-334.

Elman, J. L. (1990). Finding structure in time. Cognitive Science, 14, 179-211.

Ferreira, F. \& Patson, N. D. (2007). The 'good enough' approach to language comprehension. Language and Linguistics Compass, 1, 71-83.

Fodor, J. (1983) The modularity of mind: An essay on Faculty Psychology. Massachusetts: Bradford Books.

Frazier. (1987). Sentence processing: A tutorial review. En M. Coltheart (Ed.), Attention and perfomance XII: The psychology of reading (559-586). Nueva Jersey: Erlbaum.

Frazier, L. \& Fodor, J. D. (1978). The sausage machine: A new two-stage parsing model. Cognition, 6(4), 291-325.

Frazier, L. \& Rayner, K. (1982). Making and correcting errors during sentence comprehension: Eye movements in the analysis of structurally ambiguous sentences. Cognitive Psychology, 14(2), 178-210.

Hale, J. T. (2011). What a rational parser would do. Cognitive Science, 35, 399-443.

Hagoort, P., Brown, C. \& Groothusen, J. (1993). The syntactic positive shift (SPS) as an ERP measure of syntactic processing. Language and Cognitive Processes, 8, 439-483.

Holmqvist, K., Nystrom, M., Andersson, R., Dewhurst, R., Jarodzka, H. \& van de Weijer, J. (2011). Eye tracking: A comprehensive guide to methods and measures. Oxford: Oxford University Press.

Hyönä, J., Lorch, R., Kaakinen, J., (2002). Individual differences in reading to summarize expository text: Evidence from eye fixation patterns. Journal of Education Psychology. 94(1), 44-55. 
Hyönä, J., Lorch, R. F. \& Rinck, M. (2003). Eye movement measures to study global text processing. En J. Hyönä, R. Radach\& H. Deubel (Eds.), The mind's eye: Cognitive and applied aspects of eye movement research (pp. 313-334). Ámsterdam: Elsevier.

Irmen, L. J. (2007). What's in a (role) name? Formal and conceptual aspects of comprehending personal nouns. Psycholinguist Research, 36, 431-456.

Irmen, L. \& Roßberg, N. (2004). Gender markedness of language: The impact of grammatical and nonlinguistic information on the mental representation of person information. Journal of Language and Social Psychology, 23(3), 272-307.

Jaeger, F. T. \& Snider, N. E. (2013). Alignment as a consequence of expectation adaptation: Syntactic priming is affected by the prime's prediction error given both prior and recent experience. Cognition, 127, 57-83.

Just, M. \& Carpenter, P. (1980). A theory of reading: From eye fixations to comprehension. Psychological Review, 87(4), 329-354.

Kim, A. \& Osterhout, L. (2005). The independence of combinatory semantic processing: Evidence from event-related potentials. Journal of Memory and Language, 52, 205-225.

Kos, M., Vosse, T., van Den Brink, D., Hagoort, P., et al. (2010). About edible restaurants: Conflicts between syntax and semantics as revealed by ERPs. Frontiers in Psychology, 1, 1-11.

Kuperberg, G. R. (2007). Neural mechanisms of language comprehension: Challenges to syntax. Brain Research, 1146, 23-49.

Kuperberg, G. R., Sitnikova, T., Caplan, D. \& Holcomb, P. J. (2003). Electrophysiological distinctions in processing conceptual relationships within simple sentences. Cognitive Brain Research, 17, 117-129.

Kutas, M. \& Hillyard, S. A. (1980). Reading senseless sentences: Brain potentials reflect semantic incongruity. Science, 207, 203-205.

Kutas, M., Van Petten, C. \& Kluender, R. (2006). Psycolinguistics electrified II. En M. Traxler \& M. Gernsbacher (Eds.), Handbook of Psycholinguistics (pp. 659-724). Ámsterdam. Elsevier.

Levin, B. \& Rappaport, M. (1986). The formation of adjectival passives. Linguistic inquiry, 623-661.

Levy, R. (2008). Expectation-based syntactic comprehension. Cognition, 106, 11261177. 
Loureda, Ó., Cruz, A., Rudka, M., Nadal, L., Recio, I. \& Zuloaga, M. B. (2015). Focus particles in information processing: An experimental study on pragmatic scales with Spanish incluso. Linguistik online, 71(2).

MacDonald, M. C., Pearlmutter, N. J. \& Seidenberg, M. S. (1994). The lexical nature of syntactic ambiguity resolution. Psychological Review, 101, 483-506.

McRae, K., Ferretti, T. R. \& Amyote, L. (1997). Thematic roles as verb-specific concepts. Language and Cognitive Processes, 12(2-3), 137-176.

McRae, K. \& Matzuki, K. (2013). Constraint-based models of sentence processing. En R. van Gompel (Ed.) Sentence Processing (pp. 51-67). Londres: Psychology Press.

Mitchell, D. C. (1987). Lexical guidance in human parsing: Locus and processing characteristics. En M. Coltheart (Ed.), Attention and Performance XII (pp. 601618). Hillsdale, NJ: Erlbaum.

Mitchell, D. C. (1994). Sentence parsing. En M. Traxler \& M. Gernsbacher (Eds.), Handbook of Psycholinguistics (pp. 375-409). San Diego: Academic Press.

Moulton, J., Robinson, G. M. \& Elias, C. (1978). Sex bias in language use: 'Neutral' pronouns that aren't. American Psychologist, 33(11), 1032-1036.

Nadal, L., Cruz, A., Recio, I. \& Loureda, Ó. (2016). El significado procedimental y las partículas discursivas del español: Una aproximación experimental. Revista Signos. Estudios de Lingüistica, 49 (Supl. 1), 52-77.

Ni, W., Crain, S. \& Shankweiler, D. (1996). Sidestepping garden paths: Assessing the contributions of syntax, semantics, and plausibility in resolving ambiguities. Language and Cognitive Processes, 11, 283-334.

Parodi, G., Julio, C., Nadal, L. \& Burdiles, G. (2018). Always look back: Eye movements as a reflection of anaphoric encapsulation in Spanish while reading the neuter pronoun ello. Journal of Pragmatics, 132, 47-58.

Pickering, M. J. \& Traxler, M. J. (1998). Plausibility and recovery from garden paths: An eye-tracking study. Journal of Experimental Psychology: Learning, Memory, and Cognition, 24, 940-961.

Pickering, M. J. \& Van Gompel, R. P. (2006). Syntactic parsing. En M. Traxler \& M. Gernsbacher (Eds.), Handbook of Psycholinguistics (pp. 455-503). San Diego: Academic Press.

Recio, I., Nadal, L. \& Loureda, Ó. (2018). On argumentative relations in Spanish: Experimental evidence on the grammaticalization of cause-consequence discourse markers. Discourse Markers in Grammaticalization and Constructionalization: New Issues in the Study of Language Change. Brill, Leiden. 
Redl, T., Eerland, A. \& Sanders, T. J. (2018). The processing of the Dutch masculine generic zijn 'his' across stereotype contexts: An eye-tracking study. PloS one, 13(10), 1-22.

Speer, S. R. \& Blodgett, A. (2006). Prosody. En M. J. Traxler \& M. A. Gernsbacher (Eds.), The Handbook of Psycholinguistics (pp. 505-538). Ámsterdam: Elsevier.

Tanenhaus, M. K., Carlson, G. \& Trueswell, J. C. (1989). The role of thematic structures in interpretation and parsing. Language and Cognitive Processes, 4(3-4), 211-234.

Tanenhaus, M. K., Spivey-Knowlton, M. J., Eberhard, K. M. \& Sedivy, J. C. (1995). Integration of visual and linguistic information in spoken language. Science, 268, 1632-1634.

Tanenhaus, M. K. \& Trueswell, J. C. (1995). Sentence comprehension. En P. Eimas \& J. Miller (Eds.), Handbook in Perception and Cognition, Volume 11: Speech, Language, and Communication (pp. 217-262). San Diego, CA: Academic Press.

Traxler, M., Hoversten J. \& Trevor, A. (2018). Sentence processing and interpretation in monolinguals and bilinguals: Classical and contemporary approaches. En E. Fernández \& H. Smith (Eds.), The Handbook of Psycholinguistics (pp. 325-349). Oxford: Wiley Blackwell.

Trueswell, J. C. (1996). The role of lexical frequency in syntactic ambiguity resolution. Journal of memory and language, 35(4), 566-585.

Trueswell, J. C., Tanenhaus, M. K., \& Garnsey, S. M. (1994). Semantic influences on parsing: Use of thematic role information in syntactic ambiguity resolution. Journal of memory and language, 33(3), 285-318.

Trueswell, J. C., Tanenhaus, M. K. \& Kello, C. (1993). Verb-specific constraints in sentence processing: Separating effects of lexical preference from gardenpaths. Journal of Experimental Psychology: Learning, Memory and Cognition, 19, 528553.

Tyler, L. K. \& Marslen-Wilson, W. D. (1977). The on-line effects of semantic context on syntactic processing. Journal of Verbal Learning and Verbal Behavior, 16(6), 683-692.

van Herten, M., Kolk, H. H. \& Chwilla, D. J. (2005). An ERP study of P600 effects elicited by semantic anomalies. Cognitive Brain Research, 22, 241-255.

van Herten, M., Chwilla, D. J. \& Kolk, H. H. (2006). When heuristics clash with parsing routines: ERP evidence for conflict monitoring in sentence perception. Journal of Cognitive Neuroscience, 18, 1181-1197. 


\section{ANEXO}

\section{Estímulos críticos}

\begin{tabular}{|c|c|}
\hline Cumplimiento del estereotipo & Violación del estereotipo \\
\hline $\begin{array}{l}\text { Le compró las muñecas de porcelana a su hija. } \\
\text { Los vestidos que llevaban puestos le gustarían } \\
\text { mucho. }\end{array}$ & $\begin{array}{l}\text { Le compró las muñecas de porcelana a su hijo. Los } \\
\text { vestidos que llevaban puestos le gustarían mucho. }\end{array}$ \\
\hline $\begin{array}{l}\text { Le compró el lápiz labial a su novia. Se lo había } \\
\text { pedido desde la semana pasada. }\end{array}$ & $\begin{array}{l}\text { Le compró el lápiz labial a su novio. Se lo había } \\
\text { pedido desde la semana pasada. }\end{array}$ \\
\hline $\begin{array}{l}\text { Le dio un vestido a su hija para su cumpleaños. } \\
\text { La pequeña lo estaba pidiendo desde hace mucho } \\
\text { tiempo. }\end{array}$ & $\begin{array}{l}\text { Le dio un vestido a su hijo para su cumpleaños. El } \\
\text { pequeño lo estaba pidiendo desde hace mucho } \\
\text { tiempo. }\end{array}$ \\
\hline $\begin{array}{l}\text { La dama le cedió el asiento a su enamorado. } \\
\text { Quería hacer un gesto cortés para impresionarlo. }\end{array}$ & $\begin{array}{l}\text { La dama le cedió el asiento a su enamorada. Quería } \\
\text { hacer un gesto cortés para impresionarla. }\end{array}$ \\
\hline $\begin{array}{l}\text { Le regaló una pintura de uñas a su novia. Pasaron } \\
\text { un romántico aniversario. }\end{array}$ & $\begin{array}{l}\text { Le regaló una pintura de uñas a su novia. Pasaron un } \\
\text { romántico aniversario. }\end{array}$ \\
\hline $\begin{array}{l}\text { Ana le prestó sus mejores jeans a su prima. } \\
\text { Quería ver si le quedaban bien. }\end{array}$ & $\begin{array}{l}\text { Ana le prestó sus mejores jeans a su primo. Quería } \\
\text { ver si le quedaban bien. }\end{array}$ \\
\hline $\begin{array}{l}\text { Le entregó el ramo de flores a su novia. Después } \\
\text { de tantos años juntos aún estaba nerviosa. }\end{array}$ & $\begin{array}{l}\text { Le entregó el ramo de flores a novio. Después de } \\
\text { tantos años juntos aún estaba nerviosa. }\end{array}$ \\
\hline $\begin{array}{l}\text { Le compró una casa a su esposa el día del } \\
\text { aniversario de bodas. Llevaban } 20 \text { años juntos. }\end{array}$ & $\begin{array}{l}\text { Le compró una casa a su esposo el día del } \\
\text { aniversario de bodas. Llevaban } 20 \text { años juntos. }\end{array}$ \\
\hline $\begin{array}{l}\text { Le regaló una pelota de fútbol a su sobrino. Le } \\
\text { encantaba patear al arco }\end{array}$ & $\begin{array}{l}\text { Le regaló una pelota de fútbol a su sobrina. Le } \\
\text { encantaba patear al arco }\end{array}$ \\
\hline $\begin{array}{l}\text { Le pasó el martillo a su carpintero. El trabajo lo } \\
\text { terminó en dos días. }\end{array}$ & $\begin{array}{l}\text { Le pasó el martillo a su carpintera. El trabajo lo } \\
\text { terminó en dos días. }\end{array}$ \\
\hline $\begin{array}{l}\text { Camila le prestó los cosméticos a su hermana. } \\
\text { Quería utilizarlas para sacarse las cejas. }\end{array}$ & $\begin{array}{l}\text { Camila le prestó los cosméticos a su hermano. } \\
\text { Quería utilizarlas para sacarse las cejas. }\end{array}$ \\
\hline $\begin{array}{l}\text { Le regaló una corbata muy cara a su primo. } \\
\text { Buscaba sorprenderlo por su cumpleaños. }\end{array}$ & $\begin{array}{l}\text { Le regaló una corbata muy cara a su prima. Buscaba } \\
\text { sorprenderlo por su cumpleaños. }\end{array}$ \\
\hline
\end{tabular}

\section{NOTAS}

${ }^{1}$ La característica fundamental de la perspectiva modular corresponde a que los procesos cognitivos complejos están descompuestos en segmentos más simples, cada uno de los cuales es realizado por un subsistema especializado. De este modo, se asume que el análisis sintáctico es un proceso que ocurre luego de que el reconocimiento auditivo y/u ortográfico son completados y antes que otros procesos superiores tales como la integración discursiva o el procesamiento pragmático se hayan realizado. Además, la perspectiva modular (Frazier \& Rayner, 1982; Frazier, 1987) señala que cuando cada palabra es leída o escuchada, una primera etapa de análisis sintáctico y de procesamiento modular utiliza solo la estructura sintáctica hasta ese punto. De este modo, los factores de plausibilidad de los eventos contenidos en la oración son computados por un sistema central que opera solo cuando el análisis sintáctico de toda la oración haya sido procesado. 
${ }^{2}$ El advenimiento de la perspectiva modular es considerada, en este trabajo, exclusivamente respecto de los procesos de parsing sintáctico y no es extensivo a la perspectiva modular de la cognición en otros dominios como la organización sensorial o perceptual. 